\title{
Outcomes of patients with metastatic pancreatic cancer who progress on first restaging imaging
}

\author{
Jane E. Rogers ${ }^{1 \#}$, Jonathan D. Mizrahi ${ }^{2 *}$, Graciela M. Nogueras Gonzalez ${ }^{3}$, Rishi Surana ${ }^{2}$, \\ Rachna T. Shroff ${ }^{4}$, Robert Wolff ${ }^{5}$, Gauri R. Varadhachary ${ }^{5}$, Milind M. Javle ${ }^{5}$, Michael Overman ${ }^{5}$, \\ Kanwal Raghav ${ }^{5}$, Shubham Pant ${ }^{5,6}$
}

${ }^{1}$ Pharmacy Clinical Programs, University of Texas MD Anderson Cancer Center, Houston, TX, USA; ${ }^{2}$ Department of Cancer Medicine, University of Texas MD Anderson Cancer Center, Houston, TX, USA; ${ }^{3}$ Department of Biostatistics, University of Texas MD Anderson Cancer Center, Houston, TX, USA; ${ }^{4}$ University of Arizona Cancer Center, Tucson, AZ, USA; ${ }^{5}$ Department of Gastrointestinal Medical Oncology, University of Texas MD Anderson Cancer Center, Houston, TX, USA; ${ }^{6}$ Department of Investigation Cancer Therapeutics, University of Texas MD Anderson Cancer Center, Houston, TX, USA

Contributions: (I) Conception and design: JE Rogers, JD Mizrahi, GM Nogueras Gonzalez, S Pant; (II) Administrative support: S Pant; (III) Provision of study materials and patients: RT Shroff, R Wolff, GR Varadhachary, MM Javle, M Overman, K Raghav, S Pant; (IV) Collection and assembly of data: JE Rogers, JD Mizrahi, R Surana; (V) Data analysis and interpretation: JE Rogers, JD Mizrahi, GM Nogueras Gonzalez, S Pant; (VI) Manuscript writing: All authors; (VII) Final approval of manuscript: All authors.

\#These authors contributed equally to this work.

Correspondence to: Jane E. Rogers, Pharm.D., BCOP Clinical Pharmacy Specialist. U.T. M.D. Anderson Cancer Center, 1515 Holcombe Blvd, Unit 377, Houston, TX, USA. Email: jerogers@mdanderson.org.

Background: Objective responses to first-line systemic chemotherapy in metastatic pancreatic cancer patients are seen in less than one third of cases. Unfortunately, a significant amount will have disease progression (PD) on their first restaging imaging. With patients' short life expectancy, it is crucial for clinicians to be prudent when deciding whom and when to treat. Our study aimed to evaluate outcomes of patients that progressed on their first restaging imaging on $1^{\text {st }}$ line therapy.

Methods: We retrospectively analyzed patients diagnosed between 2010-2017 whose first restaging imaging demonstrated PD. The primary outcome was overall survival (OS) from metastatic diagnosis date to death. Patients who were lost to follow-up were excluded.

Results: Out of 262 total patients reviewed, 98 patients (37\%) were included. Sixty-five (66\%) received $2^{\text {nd }}$ line therapy, and 33 (34\%) did not. Reasons patients did not pursue $2^{\text {nd }}$ line therapy were performance status (PS) decline, organ dysfunction, or patient choice for alternative therapy. Median ages for patients who did and did not receive $2^{\text {nd }}$ line therapy were 61 and 67 , respectively $(\mathrm{P}<0.001)$. More patients had a poor PS at the time of initial diagnosis in the non- $2^{\text {nd }}$ line therapy group $(7.5 \%$ vs. $31.0 \%, \mathrm{P}=0.021)$. Median OS for those receiving $2^{\text {nd }}$ line therapy was 9 months (95\% CI: 7-11 months) compared to 4 months (95\% CI: 3-5 months) for those not receiving 2nd-line therapy $(\mathrm{P}<0.001)$.

Conclusions: Although likely biased due to better performance status and younger age, our patients who progressed rapidly on $1^{\text {st }}$ line therapy showed an OS benefit if they received $2^{\text {nd }}$ line therapy. These results suggest that patients maintaining a good PS after immediate progression on $1^{\text {st }}$ line therapy should be offered $2^{\text {nd }}$ line therapy.

Keywords: Pancreatic neoplasms; performance status; second-line therapy; carcinoma, pancreatic ductal

Submitted Dec 04, 2020. Accepted for publication Jun 08, 2021.

doi: 10.21037/jgo-20-569

View this article at: https://dx.doi.org/10.21037/jgo-20-569 


\section{Introduction}

Pancreatic ductal adenocarcinoma (PDAC) carries a poor prognosis representing the fourth most common cause of cancer-related deaths (1). By 2030, PDAC is estimated to be the second leading cause of cancer death (2). Five-year overall survival (OS) rate for those with distant disease spread is 3\% (3). Current metastatic first-line therapy is composed of two standard regimens 5-Fluorouracil $(5-\mathrm{FU})+$ irinotecan + oxaliplatin $($ FOLFIRINOX) or gemcitabine + nab-paclitaxel (1). Both regimens improved survival compared to the standard at the time gemcitabine monotherapy.

Conroy et al. established FOLFIRNOX as a front-line regimen in 2011 by conducting a phase II-III multicenter, randomized controlled trial in patients with an Eastern Cooperative Oncology Group performance status of 0 or 1 comparing standard of care gemcitabine alone to a fluoropyrimidine combination regimen of 5 -fluorouracil + leucovorin + oxaliplatin + irinotecan (FOLFIRINOX) (4). FOLFIRINOX showed both an OS and PFS advantage to gemcitabine alone (FOLFIRINOX median OS 11.1 months vs. gemcitabine median OS 6.8 months, $\mathrm{P}<0.001$; FOLFIRINOX median PFS 6.4 months $v s$. gemcitabine median PFS 3.3 months, $\mathrm{P}<0.001$ ). Progressive disease on reimaging scans in this study showed that some patients will not respond to FOLFIRINOX or gemcitabine alone (15.2\% progressive disease with FOLFIRINOX and 34.5\% progressive disease with gemcitabine alone). Von Hoff et al. established gemcitabine + nab-paclitaxel as a frontline regimen in 2013 by conducting a multicenter phase III randomized trial in patients with Karnofsky performance status score of 70 or more comparing GEM + nabpaclitaxel to gemcitabine alone in metastatic PDAC (5). The combination improved both OS and PFS (Gemcitabine + nab-paclitaxel median OS 8.5 months $v s$. gemcitabine median OS 6.7 months, $\mathrm{P}<0.001$; gemcitabine + nabpaclitaxel median PFS 5.5 months vs. GEM median PFS 3.7 months, $\mathrm{P}<0.001)$. This study additionally showed that some patients will have progressive disease on reimaging scans with gemcitabine + nab-paclitaxel or gemcitabine alone $(20 \%$ progressive disease with gemcitabine + nabpaclitaxel and $26 \%$ progressive disease with gemcitabine alone).

While disease stabilization is achievable for a significant percentage seen with FOLFIRINOX or gemcitabine + nabpaclitaxel, some patients will have radiographic evidence of disease progression on their first restaging imaging
$(4,5)$. With patients' short life expectancy in the metastatic setting, limited systemic treatment options, and significant toxicities associated with multi-drug chemotherapy, it is crucial for clinicians to be prudent when deciding whom and when to treat. The purpose of our study was to evaluate outcomes of patients who progressed on their first restaging imaging while on first-line therapy.

We present the study in accordance with the STROBE reporting checklist. Available at http://dx.doi.org/10.21037/ jgo-20-569.

\section{Methods}

Our study was a single institution, retrospective chart review of patients with metastatic PDAC who showed progression on their first restaging evaluation of firstline chemotherapy. Adult metastatic PDAC patients who were diagnosed between March 2010 to April 2017 were included. First radiographic imaging was 6-8 weeks after starting therapy. Only those patients with progressive disease on first line treatment first restaging imaging were included. Patients must have received this treatment at our center along with radiographic follow-up every 8-12 weeks at our center. Patients who received recommendations from our center but received therapy elsewhere were excluded. Patients with unresectable locally advanced disease were excluded along with those lost to follow-up. Patients who received second-line therapy were compared to those that did not receive second-line therapy. OS was the primary objective.

Data collection included patient demographics [age, gender, race, Eastern Cooperative Oncology Group (ECOG) performance status, body mass index] and tumor characteristics (metastatic disease sites, primary tumor location). Treatment factors collected were what first-line regimen and second-line regimen received. Treatment regimens given were per individual physician discretion. Reasons patients did not receive second-line therapy were also collected. CA19-9 was collected at first-line therapy start and at first-line progression. Molecular germline testing was collected when available. Additionally, date of progression and date of death were collected. Molecular analysis was collected when available.

\section{Ethical statement}

The study was conducted in accordance with the Declaration 
Table 1 Patient characteristics

\begin{tabular}{|c|c|}
\hline Characteristic & Data \\
\hline Age at diagnosis & Median 64 yo (25-84 yo) \\
\hline Baseline BMI & Median $26.1(18.1-46.1)$ \\
\hline \multicolumn{2}{|l|}{ Age } \\
\hline$<50$ yo & $7(7.1 \%)$ \\
\hline$\geq 50$ yo & $91(92.9 \%)$ \\
\hline \multicolumn{2}{|l|}{ Gender } \\
\hline Male & $58(59.2 \%)$ \\
\hline Female & $40(40.8 \%)$ \\
\hline \multicolumn{2}{|l|}{ ECOG PS at diagnosis } \\
\hline 0 & $13(15.9 \%)$ \\
\hline 1 & $56(68.3 \%)$ \\
\hline $2-3$ & $13(15.9 \%)$ \\
\hline \multicolumn{2}{|l|}{ Race } \\
\hline Caucasian & $73(74.5 \%)$ \\
\hline Non-Caucasian & $25(25.5 \%)$ \\
\hline \multicolumn{2}{|l|}{ Metastatic disease sites } \\
\hline Diffuse & $55(56.1 \%)$ \\
\hline Single organ & $43(43.9 \%)$ \\
\hline \multicolumn{2}{|l|}{$1^{\text {st }}$ Line therapy } \\
\hline FOLFIRINOX & $34(34.7 \%)$ \\
\hline Gemcitabine + nab-paclitaxel & $57(58.2 \%)$ \\
\hline Gemcitabine + cisplatin & $2(2.0 \%)$ \\
\hline $5-\mathrm{FU}+/-$ oxaliplatin & $1(1.0 \%)$ \\
\hline Gemcitabine +/- erlotinib & $3(3.1 \%)$ \\
\hline Trial & $1(1.0 \%)$ \\
\hline \multicolumn{2}{|l|}{ CA19-9 at diagnosis } \\
\hline$<35$ & $9(10.3 \%)$ \\
\hline$<59 \times$ ULN & $38(43.7 \%)$ \\
\hline$>59 \times$ ULN & $40(46.0 \%)$ \\
\hline \multicolumn{2}{|l|}{ Second-line therapy received } \\
\hline Yes & $65(66.3 \%)$ \\
\hline No & $33(33.7 \%)$ \\
\hline \multicolumn{2}{|l|}{ CA19-9 at first-line progression } \\
\hline$<35$ & $8(8.9 \%)$ \\
\hline$<59 \times$ ULN & $34(37.8 \%)$ \\
\hline$>59 \times$ ULN & $48(53.3 \%)$ \\
\hline
\end{tabular}

Table 1 (continued)
Table 1 (continued)

\begin{tabular}{lc}
\hline Characteristic & Data \\
\hline Second-line therapy & $33(33.7 \%)$ \\
None & $31(31.6 \%)$ \\
Gemcitabine + nab-paclitaxel & $8(8.2 \%)$ \\
FOLFIRINOX & $8(8.2 \%)$ \\
$5-F U+/-$ oxaliplatin & $3(3.1 \%)$ \\
$5-F U+$ irinotecan or liposomal & $12(12.3 \%)$ \\
irinotecan & $3(3.1 \%)$ \\
Gemcitabine based therapy & \\
Other & $18(27.7 \%)$ \\
Second-line therapy radiographic results & $47(72.3 \%)$ \\
Disease-control & \\
Progression & \\
\hline ECOG, Eastern Cooperative Oncology Group; PS, performance \\
status.
\end{tabular}

of Helsinki (as revised in 2013). Our institutional review board approved our study (PA16-0738). A waiver of consent was granted given the minimal risk of a retrospective evaluation.

\section{Statistical analysis}

The objectives of this study were to describe the study population overall and by second-line chemotherapy, and overall survival (OS). The Kaplan-Meier product limit method (Kaplan \& Meier, 1958) was used to estimate the median OS. Univariate Cox proportional hazards regression was used to identify any association with each of the variables and OS. Statistical analysis was performed using Stata/SE version 16.0 statistical software (Stata Corp. LP, College Station, TX, USA).

\section{Results}

Ninety-eight patients were included with a median age of 64 years old. Median follow-up for the entire group was 6.8 months. Patient characteristics are described in Table 1. Most patients did not have germline testing performed (93\%). One patient had BRCA1 7990del3ins2. The remainder were negative for BRCA1 and 2. Sixty-five patients received second-line therapy while 33 patients did not receive second-line therapy. Median first-line 
PFS for those that did receive second-line therapy was 1.84 months (95\% CI: 1.81-2.01 months), and median first-line PFS for those that did not receive second-line therapy was 1.48 months (95\% CI: 1.12-1.71 months). Reasons for not pursuing second-line therapy were PS decline due to disease complications $(48 \%, \mathrm{n}=16)$, patient's choice to stop or pursue alternative medicine $(24 \%, n=8), 5$ PS decline due to other comorbidities such as dementia, congestive heart failure, chronic kidney disease $(15 \%, n=5)$, organ dysfunction not suitable for second-line therapy options $(6 \%, n=2)$, or poor PS due to chemotherapy toxicity $(6 \%, \mathrm{n}=2)$.

Patient characteristics between the two groups are summarized in Table 2. Factors statistically different between groups were age, ECOG PS at diagnosis, and first-line regimen. Patients were younger in the population that received second-line therapy (median age 61 years old) compared to those that did not receive second-line therapy (median age 67 years old, $\mathrm{P}<0.001$ ). More patients had an ECOG PS of 2-3 at baseline in the non-second-line therapy group $(7.5 \%$ in second-line therapy group $v s .31 \%$ in non-second line therapy group, $\mathrm{P}=0.021)$. Forty-five percent received FOLFIRNOX front-line in the secondline therapy group compared to $15 \%$ in the non-secondline therapy group. Forty-five percent in the second-line therapy group received gemcitabine + nab-paclitaxel frontline. Most patients in the non-second-line therapy group received gemcitabine + nab-paclitaxel first-line. Those that had disease-control with second-line therapy received gemcitabine + nab-paclitaxel $(n=8)$, FOLFIRINOX $(n=3)$, FOLFOX/5-FU ( $n=1)$, or gemcitabine-based therapy $(n=6)$. Median OS was 9 months in the second-line therapy group compared to 4 months in the non-second-line therapy group, $\mathrm{P}<0.001$ (Figure 1).

\section{Discussion}

PDAC remains a challenging cancer to treat with unfortunately limited progress in management. Less than a decade ago, front-line therapy with FOLFIRINOX and gemcitabine + nab-paclitaxel were determined to be preferred regimens $(1,4,5)$. However, almost half of patients remain in good condition following front-line therapy, yet second-line therapy is less defined (6). Limited data shows a potential survival benefit with second-line therapy (6-8). Unfortunately, these regimens studied prospectively in the second-line setting were following therapy that is no longer the preferred front-line treatment. Therefore, challenges exist with determining who will likely benefit from secondline therapy and with what second-line therapy.

Vienot et al. performed a prospective populationbased cohort study in hopes of developing a prognostic nomogram and score that would predict OS from the start of second-line therapy (6). Three hundred ninetyfive patients who had received front-line advanced PDAC therapy were eligible for medical evaluation for second line therapy. Sixty-six percent of patients $(n=261)$ were treated with second-line therapy while $33.9 \%(n=134)$ did not received treatment second-line. Our study showed similarities in that most patients $(66 \%)$ pursued secondline therapy. Vienot et al. found older age, worse ECOG performance status, and shorter duration of front-line therapy were found in the untreated second-line therapy group. The authors reported age, smoking status, presence of liver metastases, performance status, pain, jaundice, ascites, duration of front-line treatment, and secondline regimen were identified as independent prognostic factors for OS with second-line therapy. Models like that established by Vienot et al. are the start of establishing a clinical guide to making decisions after front-line therapy progression.

We recognize the limitations of our retrospective review as there were significant differences between our comparator group including older age and poorer ECOG PS in the group that did not receive second-line chemotherapy. However, our results did suggest that if patients maintained a good ECOG PS after front-line fast progression, patients had improved OS if they received second-line therapy. A specific second-line therapy for this population was unable to be determined given the small numbers of our study and variety of regimens received. We believe these patients should be offered second-line therapy. Our data collection was prior to national recommendations for all patients to receive germline testing. Therefore, no conclusions could be made regarding outcomes based off genetics. 
Table 2 Comparison between second-line chemotherapy group and non-second-line chemotherapy group

\begin{tabular}{|c|c|c|c|c|c|}
\hline Characteristic & \multicolumn{2}{|c|}{ Yes } & \multicolumn{2}{|c|}{ No } & $P$ value \\
\hline Age at met diagnosis & & & & & $<0.001$ \\
\hline $\mathrm{N}$ & \multicolumn{2}{|c|}{65} & \multicolumn{2}{|c|}{33} & \\
\hline Mean (SD) & \multicolumn{2}{|c|}{$61(10.0)$} & \multicolumn{2}{|c|}{$68(10.0)$} & \\
\hline Baseline BMI or height and weight at baseline & & & & & 0.916 \\
\hline $\mathrm{N}$ & \multicolumn{2}{|c|}{65} & \multicolumn{2}{|c|}{33} & \\
\hline Mean (SD) & \multicolumn{2}{|c|}{$26.88(4.57)$} & \multicolumn{2}{|c|}{$27.36(5.84)$} & \\
\hline Median [Min-Max] & \multicolumn{2}{|c|}{26.10 [18.10-39.10] } & \multicolumn{2}{|c|}{$26.90[20.40-46.10]$} & \\
\hline Mean (SD) & \multicolumn{2}{|c|}{$10.11(6.29)$} & \multicolumn{2}{|c|}{$4.73(3.33)$} & \\
\hline Median (Min-Max) & \multicolumn{2}{|c|}{$8.51(3.16-38.05)$} & \multicolumn{2}{|c|}{$3.88(1.88-19.29)$} & \\
\hline Age & & & & & 0.418 \\
\hline$<50$ & 6 & 9.23 & 1 & 3.03 & \\
\hline$\geq 50$ & 59 & 90.77 & 32 & 96.97 & \\
\hline Gender & & & & & 0.125 \\
\hline Male & 42 & 64.62 & 16 & 48.48 & \\
\hline Female & 23 & 35.38 & 17 & 51.52 & \\
\hline No & 22 & 33.85 & 13 & 39.39 & \\
\hline Yes & 43 & 66.15 & 20 & 60.61 & \\
\hline White & & & & & 0.837 \\
\hline No & 48 & 73.85 & 25 & 75.76 & \\
\hline Yes & 17 & 26.15 & 8 & 24.24 & \\
\hline Site of disease & & & & & 0.437 \\
\hline Head & 31 & 47.69 & 19 & 57.58 & \\
\hline Body & 19 & 29.23 & 10 & 30.30 & \\
\hline Tail & 15 & 23.08 & 4 & 12.12 & \\
\hline
\end{tabular}

Table 2 (continued) 
Table 2 (continued)

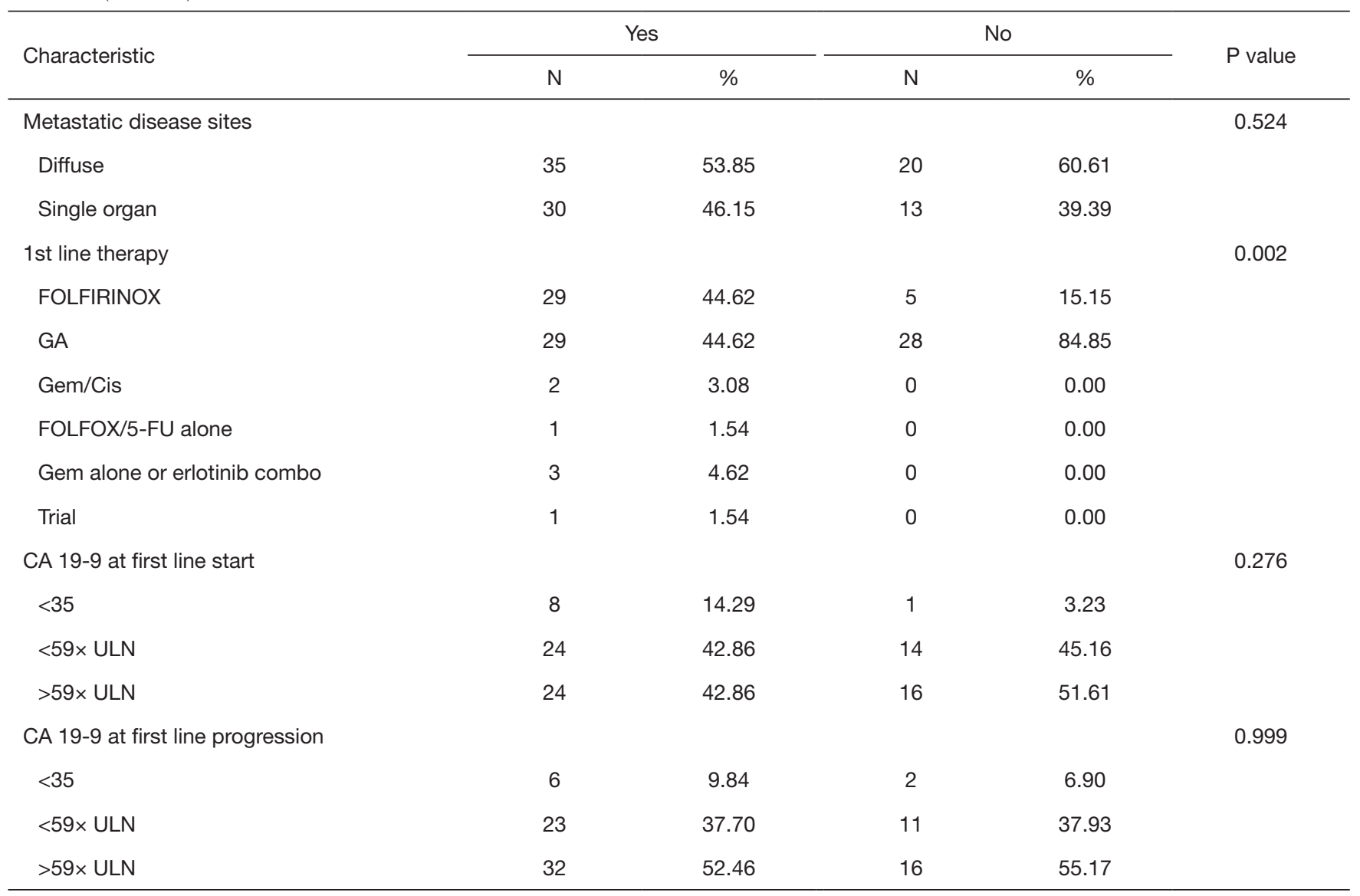

Second line therapy: N (events) Median (95\% Cl)

- Yes: 65 (65) 9 months (7-11)

- - - No: 33 (33) 4 months (3-5)

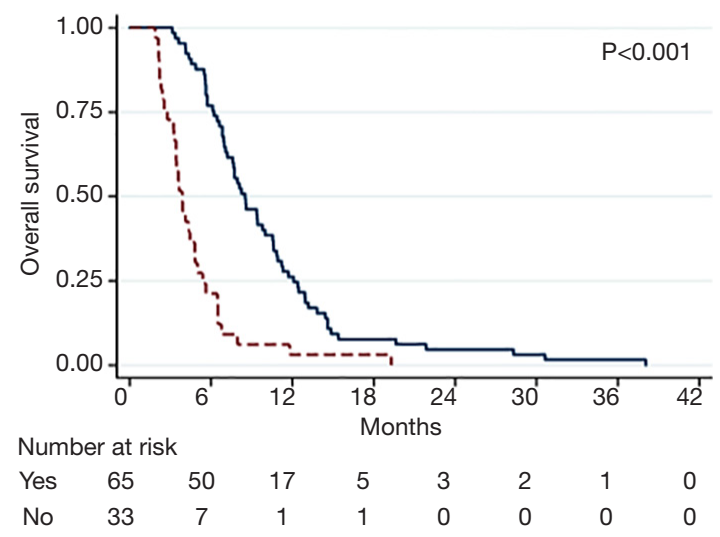

Figure 1 Overall survival comparison: second-line therapy group versus non-second line therapy group.

\section{Acknowledgments}

Funding: None.

\section{Footnote}

Reporting Checklist: The authors have completed the STROBE reporting checklist. Available at http://dx.doi. org/10.21037/jgo-20-569

Data Sharing Statement: Available at http://dx.doi. org/10.21037/jgo-20-569

Conflicts of Interest: All authors have completed the ICMJE uniform disclosure form (available at http://dx.doi. org/10.21037/jgo-20-569). MMJ currently serves as unpaid editorial board member of the fournal of Gastrointestinal 
Oncology from Jan 2021-Dec 2022. The other authors have no conflicts of interest to declare.

Ethical Statement: The authors are accountable for all aspects of the work in ensuring that questions related to the accuracy or integrity of any part of the work are appropriately investigated and resolved. The study was conducted in accordance with the Declaration of Helsinki (as revised in 2013). Our institutional review board approved our study (PA16-0738). A waiver of consent was granted given the minimal risk of a retrospective evaluation.

Open Access Statement: This is an Open Access article distributed in accordance with the Creative Commons Attribution-NonCommercial-NoDerivs 4.0 International License (CC BY-NC-ND 4.0), which permits the noncommercial replication and distribution of the article with the strict proviso that no changes or edits are made and the original work is properly cited (including links to both the formal publication through the relevant DOI and the license). See: https://creativecommons.org/ licenses/by-nc-nd/4.0/.

\section{References}

1. National Comprehensive Cancer Network. Pancreatic Adenocarcinoma. Version 1.2021. Accessed 10/28/2020.

Cite this article as: Rogers JE, Mizrahi JD, Nogueras Gonzalez GM, Surana R, Shroff RT, Wolff R, Varadhachary GR, Javle MM, Overman M, Raghav K, Pant S. Outcomes of patients with metastatic pancreatic cancer who progress on first restaging imaging. J Gastrointest Oncol 2021;12(5):2268-2274. doi: 10.21037/jgo-20-569
2. McGuigan A, Kelly P, Turkington RC, et al. Pancreatic cancer: A review of clinical diagnosis, epidemiology, treatment and outcomes. World J Gastroenterol 2018;24:4846-61.

3. National Cancer Institute. Surveillance, Epidemiology, and End Results Program. Cancer Stat Facts: Pancreatic Cancer. Accessed 10/28/2020.

4. Conroy T, Desseigne F, Ychou M, et al. FOLFIRINOX versus gemcitabine for metastatic pancreatic cancer. $\mathrm{N}$ Engl J Med 2011;364:1817-25.

5. Von Hoff DD, Ervin T, Arena FP, et al. Increased survival in pancreatic cancer with nab-paclitaxel plus gemcitabine. N Engl J Med 2013;369:1691-703.

6. Vienot A, Beinse G, Louvet C, et al. Overall Survival Prediction and Usefulness of Second-Line Chemotherapy in Advanced Pancreatic Adenocarcinoma. J Natl Cancer Inst 2017. doi: 10.1093/jnci/djx037.

7. Pelzer U, Schwaner I, Stieler J, et al. Best supportive care (BSC) versus oxaliplatin, folinic acid and 5 -fluorouracil (OFF) plus BSC in patients for second-line advanced pancreatic cancer: a phase III-study from the German CONKO-study group. Eur J Cancer 2011;47:1676-81.

8. Wang-Gillam A, Li CP, Bodoky G, et al. Nanoliposomal irinotecan with fluorouracil and folinic acid in metastatic pancreatic cancer after previous gemcitabine-based therapy (NAPOLI-1): a global, randomised, open-label, phase 3 trial. Lancet 2016;387:545-57. 